\title{
Fractional CO2 Laser Treatment after Transvaginal Polypropilene Mesh for Urinary Incontinence and Pelvic Organ Prolapse Treatment as an Alternative to Improove Mesh Erosion Rates
}

\section{Gonzalez Pablo}

Head Chief Urogynecology and Minimally Invasive Surgery

Department of Obstetrics and Gynecology, Hospital Universitario San Jorge, Pereira Colombia

*Corresponding author: Gonzalez Pablo; pagonza@ hotmail.com

Received 17 November 2021;

Accepted 27 November 2021;

Published 06 December 2021

\begin{abstract}
The support advantages of synthetic mesh in vaginal reconstructive surgery and stress urinary incontinence are significant. However, erosion of the mesh may occur as a complication, Four sessions of fractional $\mathrm{CO}^{2}$ Laser treatment protocol (Mona Lisa Touch Deka M.E.L.A Florence Italy protocol, were performed in the study group to 96 patients, beginning at 30 days, after mesh insertion, important improvement regarding erosion rates was found inside our study group, thanks to the trophic effects of fractional $\mathrm{CO}^{2}$ laser protocol. The use of alternative technologies that provide bio -stimulation of vaginal epithelium by increasing its thickness, and trophic changes that may be related to the very low erosion rate was found in our group of patients
\end{abstract}

Keywords: Urinary Incontinence, Fractional $\mathrm{CO}_{2}$ Laser, Vaginal Mesh, pelvic organ prolapse

\section{Introduction}

The support advantages of synthetic mesh in vaginal reconstructive surgery and stress urinary incontinence are significant. However, erosion of the mesh may occur as a complication

Our AIM was to improve the erosion rate by bio Stimulating vaginal trophism after fractional $\mathrm{CO}_{2}$ Laser protocol Mona Lisa touch Deka M.E.L.A Florence Italy in our select group of patients undergoing vaginal stress incontinence and prolapse surgeries using synthetic mesh.

The use of fractional $\mathrm{CO}_{2}$ laser has been described in the literature regarding its trophic effects at the level of the vaginal epithelium, the most important findings were modulation of metalloproteinases and new collagen and elastin formation ${ }^{[1,2,3]}$.

Previous report in the literature have described similar findings regarding the use of fractional $\mathrm{CO}_{2}$ Laser, as an alternative treatment for patients facing polypropylene mesh erosion ${ }^{[4]}$.

\section{Materials and Methods}

96 women between 45 and 78 years, mean age 61,83 underwent transvaginal and laparoscopic vaginal reconstructive surgery with synthetic mesh between January 2015 and January 2018 mean parity status was 3,0,69,7\% were sexually active $30,3 \%$ were nonsexually active The indications were for the treatment of stress urinary incontinence with or without pelvic organ prolapse repair. All patients in the study period had 3 years follow-up. Procedures were subdivided into four groups: 50 patients underwent a polypropylene TVT (Vertical Bottom-Top) sling 25 patients underwent a Transvaginal needless free trans obturator polypropylene mesh(Uphold capio system Boston Scientific) for anterior and apical repair, 8 patients underwent a polypropylene TOT (Horizontal Out-In, and 13 underwent Laparoscopic sacrocolpopexy (upsylon mesh with colpo assist system Boston scientific), in which mesh was used to support the apex as well as the other compartments. Four sessions of fractional $\mathrm{CO} 2$ Laser treatment protocol (Mona Lisa Touch Deka M.E.L.A Florence Italy protocol, were performed in the study group beginning at 30 days ,after mesh insertion, and subsequently at 4-6 week interval, total number of sessions 4 ,Parameters used Watt 40 Dwell time 1100 Spacing 600 Stack 2 Deka pulse (DP),punch biopsies were performed inside vaginal walls, before and after the treatment protocol, sent to the pathology department for evaluation with different staining methods .Fig 4-5 


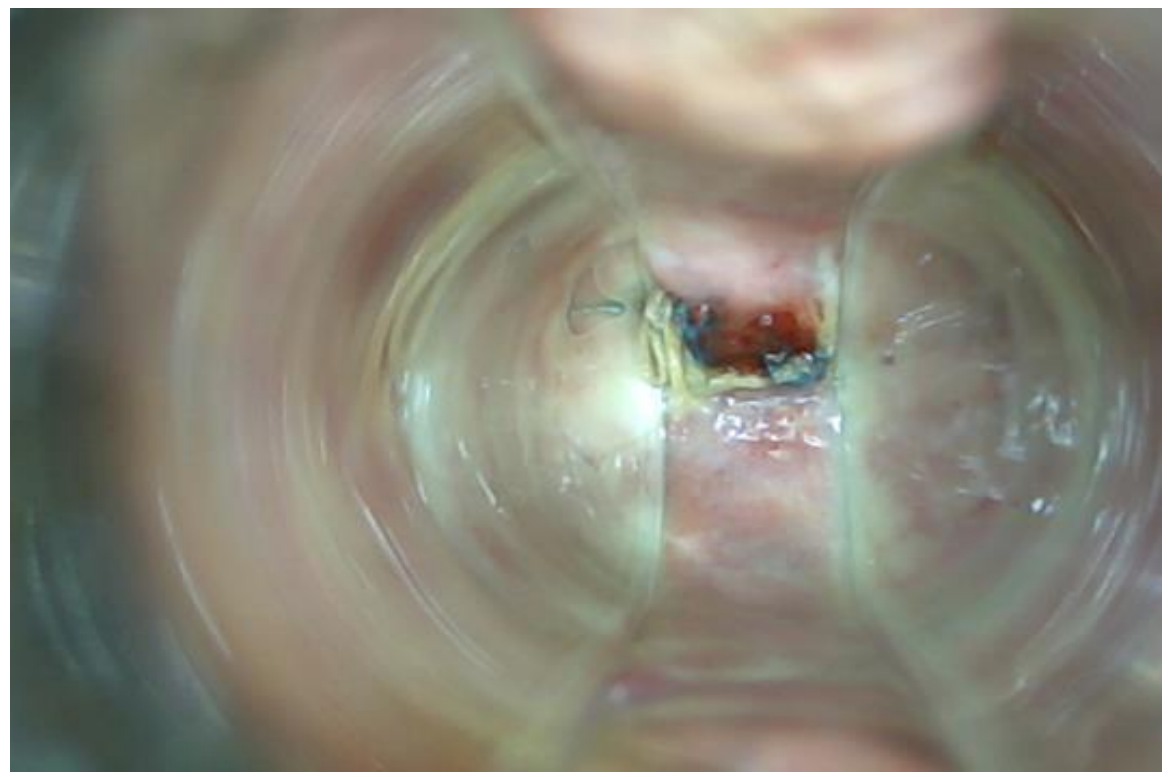

Fig 1.Upsylon/colpoassits (laparoscopic sacrocolpopexy mesh erosion 40 days)

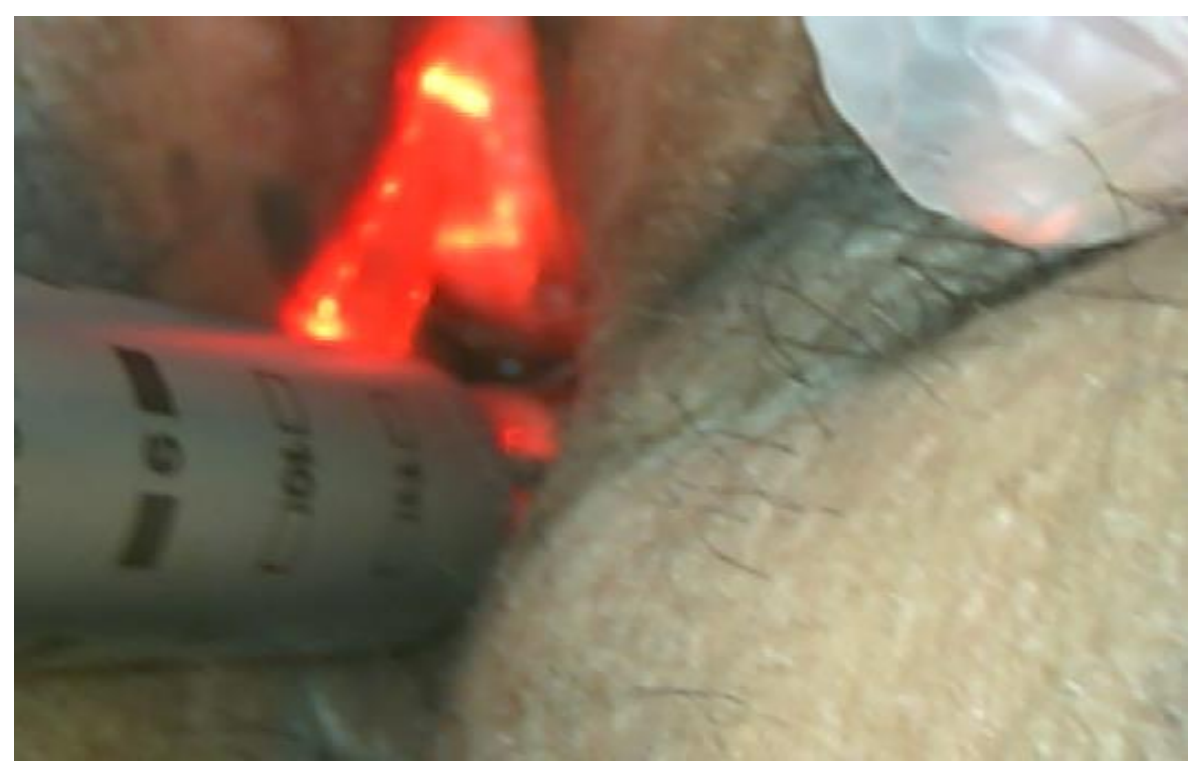

Fig 2: Fractional CO2 Laser treatment protocol (Mona Lisa Touch Deka M.E.L.A Florence Italy.

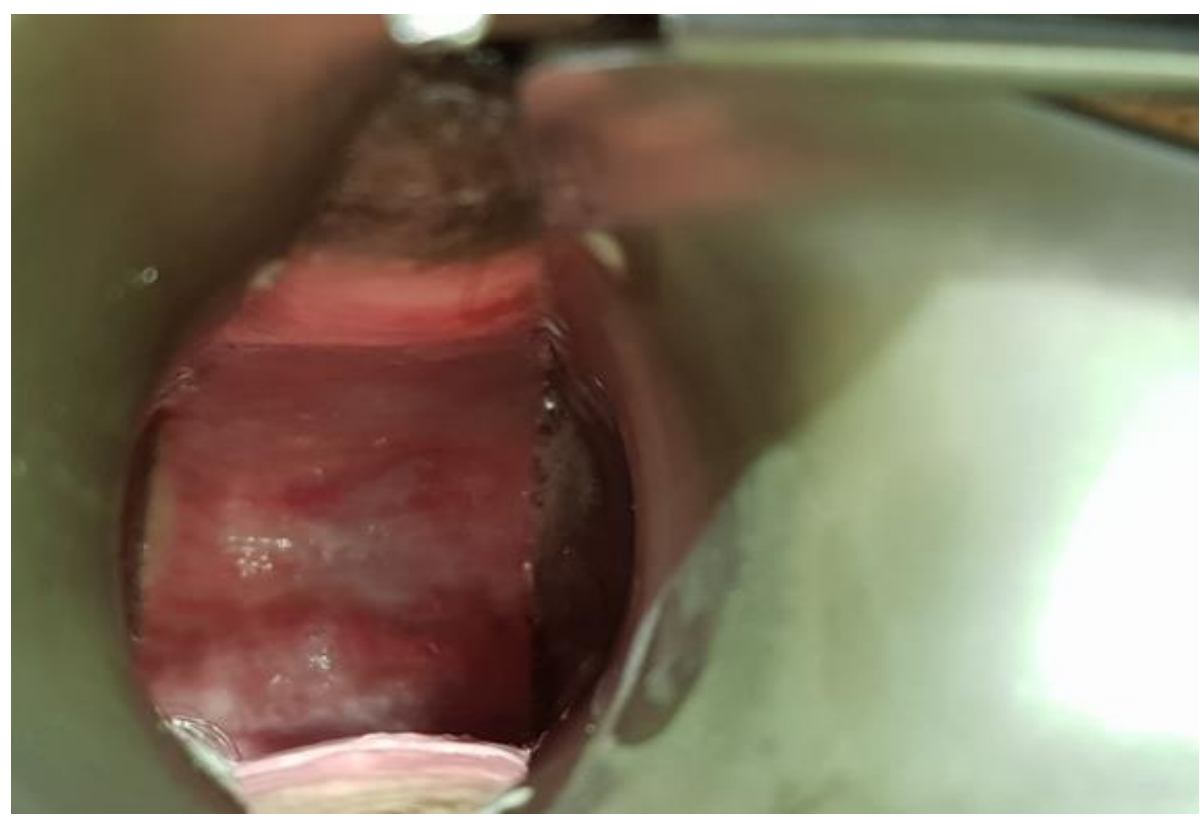

Fig 3: Complete reepithelization after 4 sessions of Fractional CO2 Laser treatment protocol (Mona Lisa Touch Deka M.E.L.A Florence Italy 


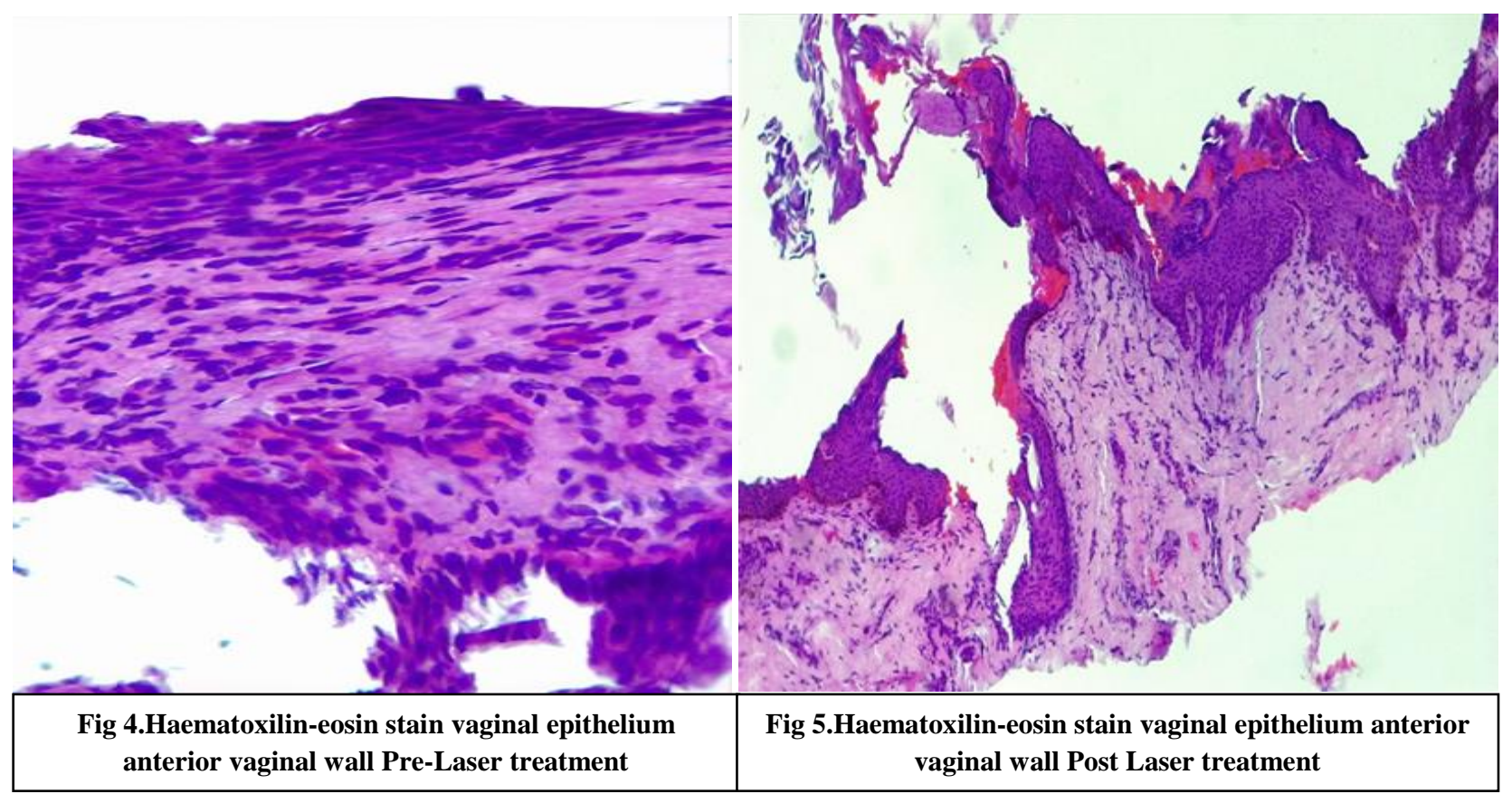

\section{Results}

Overall, mesh erosion was observed in 3 of the 96 patients (3.1\%).The rate of mesh erosion was $8,1 \%(2 / 25)$ in the Transvaginal needless free trans- obturator polypropylene mesh(Uphold capio system Boston Scientific) for anterior and apical repair group, $7,6 \%(1 / 13)$ in the Laparoscopic sacrocolpopexy (upsylon mesh with colpo assist system Boston scientific group $0 \%(0 / 50)$ in the TVT (Vertical Bottom-Top) group, also erosion free at three-year post-op was $100 \%$. The range to mesh erosion in the up hold and laparoscopic sacrocolpopexy was forty days. Erosion was noticed upon vaginal examination before the first session of the laser protocol, it did not interrupt the planned protocol.

Table 1: Erosion rates, between mesh insertion groups.

\begin{tabular}{|l|l|l|l|}
\hline Type Of Surgery & Patients & Erosion Rate & Time To Erosion \\
\hline Tvt & 50 & $0 \%$ & \\
\hline Tot & 25 & $0 \%$ & \\
\hline Uphold Trans Obturator Needless Free Capio System & 8 & $8.1 \%(2-25)$ & 40 Days \\
\hline Upsylon/Colpoassist Laparoscopic Sacrocolpopexy & 13 & $7,6 \%(1-13)$ & 40 Days \\
\hline
\end{tabular}

\section{Discussion}

There is an approximate erosion rate of mesh repairs for stress urinary incontinence in combination with anterior, posterior and/or apical prolapse of about $11 \%$ our patients sustained lower vaginal and/or urinary tract mesh erosion at 36 month follow up, We believe that the trophic effects of fractional $\mathrm{CO}_{2}$ Laser protocol was responsible to keep the vast majority of our study group patients erosion free, also patients with erosion healed after fractional $\mathrm{CO}_{2}$ Laser Application .current investigations, found diverging effects in the epithelial and stromal compartments related to decreased collagen content and decreased expression of transcripts for extracellular contents with topical estrogen therapy administration in the acute injured vagina after surgery, therefore acute administration of vaginal estrogen may have adverse effects on the early phase of healing of the fibro muscular layer despite its positive effects on the epithelium ${ }^{[5]}$, moreover the trophic effects of fractional $\mathrm{CO}_{2}$ laser demonstrated important ultra-structural modulation of Metalloproteinase, which are collagenases that are related to the balance of fibroblast production/degradation, the increase of MMP-2 active form and the corresponding decrease of the latent form in the biopsy 1 hour following treatment, with fractional $\mathrm{CO}_{2}$ laser is constituting a significant indication of production and degradation of collagen, as the stimulation of a new equilibrium occurring in the regenerative process of the connective tissue $^{[6]}$.

\section{Conclusions}

Our investigation provides a large series of prolapse and urinary incontinence cases done by a single surgeon with follow-up that extends out for three years. The use of alternative technologies that provide bio -stimulation of vaginal epithelium by increasing its thickness, and trophic changes that may be related to the very low erosion rate was found in our group of patients, As more experienced pelvic surgeons adopt the use of this novel techniques, we should be able to reduce the risk of erosion even further.

\section{Ethics approval and consent to participate}

Patients recruited for this study were volunteers from the Urogynaecology unit. They offered to participate prior to obtaining informed consent and a written authorization to be included in their medical records according to Helsinki declaration, Belmont report, CIOMS rules, GPC/ICH and 008430 resolution of Colombia government stabi lized in 4th October 1993. Present investigation was considering as minimal risk or beyond minimal risk as follows: - Adjust and briefly explain the ethical principles that warrant investigation according to international standards. - 
Based on previously conducted experiments on animals in laboratories and other scientific facts that show a secure intervention in humans. - Clearly express the risks and security guarantees to participants. - Having the written informed consent of research subjects or their legal representative. - Relate the experience of researchers and the responsibility of a health entity.

\section{List of abbreviations}

CO2 LASER(Carbon Dioxide Laser)

TVT Retropubic, vertical medio urethral sling

TOT Trans obturator,horizontal medio uretharl sling

MMP2 Metalloproteinases

\section{Data Availability}

Readers can access the data underlying the findings of the study, writing directly to corresponding author.

\section{Conflicts of Interest}

Author declares that there is no conflict of interest regarding the publication of this paper."

\section{Funding Statement}

No funding received by any any institution.

\section{Authors' contributions}

All the paper is a responsibility of the main author

\section{Bibliography}

[1] Pablo Gonzalez Isaza,Kinga Jajaguszewska,mariusz lutkazuk,jose Luis Cardona,Long -term effect, of thermoablative Fractional $\mathrm{CO} 2$ Laser treatment as a novel approach, to urinary incontinence management in women with genitourinary syndrome of menopause,Interna Urogyn Jour 2017 June

[2] Fractional $\mathrm{CO} 2$ laser effect on thick connective tissue of the vaginal wall of women with anterior vaginal prolapse: an ex-vivo study Stefano Salvatore 1,*, S. Virgilio 2, S. Palmieri1, S.Girardelli 1, A. Redaelli 1, M. Parma1, M.Candiani1, A. Calligaro 31 Vita-Salute San Raffaele University, IRCCS San Raffaele Hospital,
Milan, Italy Department of Molecular Medicine, Biochemistry Unit, University of Pavia, Pavia, Italy Department of Public Health, Experimental and Forensic Medicine, Histology and Embryology Unit, University of Pavia, Pavia, Italy

[3] Zerbinati N, Serati M, Origoni M, Candiani M, Iannitti T, Salvatore S, Marotta F, Calligaro A (2015) Microscopic and ultrastructural modifications of postmenopausal atrophic vaginal mucosa after fractional carbon dioxide laser treatment. Lasers Med Sci 30:429436

[4] Steinberg BJ, Mapp T, Mama S, Echols KT. Surgical Treatment of Persistent Vaginal Granulation Tissue Using CO2 Laser Vaporization under Colposcopic and Laparoscopic Guidance. JSLS : Journal of the Society of Laparoendoscopic Surgeons. 2012;16(3):488-491. doi:10.4293/108680812X13462882736619.

[5] Riperda.C et all .Vaginal estrogen, a dual -edged sword in post-operative healing of the vaginal wall Menopause: The Journal of The North American Menopause Society Vol. 24, No. 7, pp. 838-849

[6] Salvatore. S. et all. Early regenerative modifications of human postmenopausal atrophic vaginal mucosa following fractional CO2 laser treatment In print ,Open Access Macedonian Journal of Medical Sciences

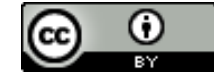

Open Access This article is licensed under a Creative Commons Attribution 4.0 International License, which permits use, sharing, adaptation, distribution and reproduction in any medium or format, as long as you give appropriate credit to the original author(s) and the source, provide a link to the Creative Commons license, and indicate if changes were made. The images or other third party material in this article are included in the article's Creative Commons license, unless indicated otherwise in a credit line to the material. If material is not included in the article's Creative Commons license and your intended use is not permitted by statutory regulation or exceeds the permitted use, you will need to obtain permission directly from the copyright holder. To view a copy of this license, visit https://creativecommons.org/licenses/by/4.0/.

(C) The Author(s) 2021 